\title{
Evaluating the Real-world Benefits and Risks of Anti-Tumor Necrosis Factor Therapies
}
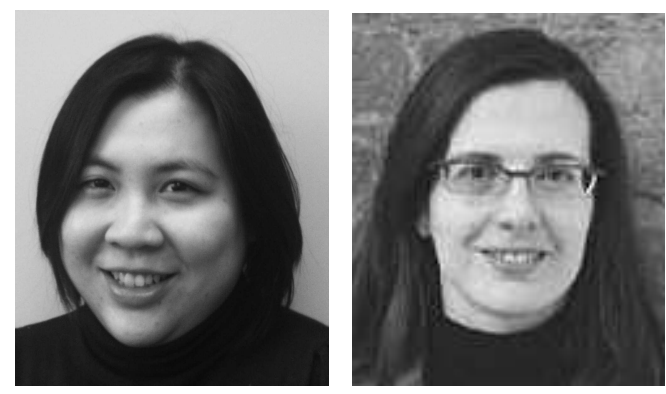

The discovery of tumor necrosis factor- $\alpha$ (TNF- $\alpha)$ as the pivotal cytokine in the inflammatory pathway and the development of drugs specifically targeted at this molecule has revolutionized our treatment of patients with rheumatoid arthritis (RA) and other inflammatory arthritides over the past decade. Trial after trial, investigators have consistently demonstrated efficacy in reducing disease activity, inflammation, and radiographic progression with anti-TNF therapy ${ }^{1}$. However, questions remain regarding the longterm safety of anti-TNF therapy because of the pleiotropic effects of TNF- $\alpha$ in immune system regulation. Such questions cannot always be answered in the context of a randomized controlled trial (RCT). By its very nature, an RCT is an experiment, an attempt to replicate a controlled laboratory environment using a homogeneous population of study subjects, with preset questions. This limits the generalizability of the results to the wider population of patients we see in routine clinical practice ${ }^{2}$. Also, some questions, especially about longterm safety, cannot always be answered because the duration of an RCT is relatively short. Observational studies can go some way toward answering these pertinent questions.

Over the past decade, a number of biologic registers and other observational studies of "real-world" anti-TNF use have been established and have started to address important questions regarding treatment benefits ${ }^{3,4}$, longterm treatment persistence ${ }^{5}$, and safety, with a focus on serious infections $6,7,8$ and malignancy ${ }^{9,10,11}$. Many of these studies have been based in large healthcare claims databases, which gather details about patient interactions with the healthcare system $^{12}$. These studies have the advantage of large sample sizes but can be limited in the detail they contain regarding other treatments and comorbidities that may be acting as confounders in the assessment of risk between treated and untreated patients.

In this issue of the Journal, Zisman, et al present the results of a retrospective study examining the occurrence of hospitalizations in 333 patients before and after commencement of anti-TNF therapy ${ }^{13}$. The study was based on a computerized health insurance database covering about half ( $\mathrm{n}>1$ million) of the total population in northern Israel. Subjects with a physician diagnosis of RA, psoriatic arthritis (PsA), and ankylosing spondylitis (AS) were included, with RA accounting for about $60 \%$ of the study group. Each patient acted as his/her own control, with the period of taking anti-TNF therapy as the "case" and the corresponding length of time immediately prior to starting anti-TNF therapy as the "control" period. Anti-TNF drugs in this analysis were etanercept, infliximab, and adalimumab, analyzed as a single group. The outcome of interest was the occurrence of hospital admission from any cause and the reason for the admission. Hospital admissions were reviewed by 2 physicians according to a protocol.

The total analysis time was 57 months. Overall, the authors found that the number of hospitalizations decreased in the period patients received anti-TNF therapy (total 300 admissions) compared to the period prior to commencing anti-TNF therapy (total 435 admissions). This decrease was largely driven by a reduction in admission for rheumatic disease flares after commencing anti-TNF therapy, in keeping with the known effectiveness of these drugs. This is an important observation and adds to the economic benefits of these therapies. Reducing the rate of hospitalization could cut costs for the health service as well as the wider economy. Hospital admissions are costly and work productivity declines when patients have to take leaves of absence for illness. These friction costs are often excluded from health economic models, which are focused on calculation of quality-adjusted life-years (QALY) based on changes in health utility measures, such as the EuroQol-5D $\mathrm{D}^{14,15}$.

While the rate of hospitalizations for exacerbations of disease was reduced, an increase in the rate of hospitalizations for infection was also observed in the period during

\section{See Hospitalizations of patients receiving anti-TNF- $\alpha$ therapy, page 16}


anti-TNF therapy compared to the period before. This is consistent with research from other prospective observational studies, which found a small but significant increase in the overall rate of serious infections (defined as infections resulting in death or hospitalization) with anti-TNF therapies $6,7,16$. This finding would be in keeping with the known biological effects of anti-TNF therapies. The rate of infection is not constant over time, however. In the British Society for Rheumatology Biologics Register, an increase in infection risk was seen, especially in the first 6 months ${ }^{6}$, with a decline thereafter. This risk appears to decline with time for several reasons: physicians may have ceased anti-TNF therapy in those patients who experienced an infection and did not restart anti-TNF therapy because of concerns about further infection, thus depleting the pool of susceptible persons. Other reasons are a reduction in steroid use and an improvement in disability and physical function ${ }^{17,18}$.

The majority of studies to date looking at the risk of serious infection have compared an anti-TNF-treated group with a separate group of patients starting or receiving a nonbiologic disease-modifying antirheumatic drug (nbDMARD), usually methotrexate. In doing so, it is possible that some of the observed increase in risk may be explained by channelling bias or confounding by indication. Anti-TNF therapy tends to be reserved for patients with the most severe disease. These patients are also more likely to be at an increased risk of serious infection because of higher use of corticosteroids and more severe disability, both recognized risk factors for infection. There may be other factors that influence a physician's decision to treat with anti-TNF that cannot be measured but may also influence a patient's risk of infection. Therefore, careful consideration of these confounders is necessary when analyzing the risk of adverse events to minimize the effects of this bias.

To overcome this, the authors of the current study used a study design in which each patient acted as his/her own control. This has the benefit of controlling for some of the unmeasured confounders, because these should be similar in the patient before and after treatment (for example, diabetes, smoking, and preexisting cardiorespiratory disease). However, this design may not overcome the effects of selection bias for deciding when and in whom to initiate treatment. The decision to start anti-TNF therapy is not made at random but is decided between doctor and patient at the time it is felt to be most beneficial. As discussed, anti-TNF treatment is usually reserved for patients with the most severe disease. This may have been characterized in this cohort by patients requiring increasing admissions for arthritis disease activity, perhaps representative of recurrent flares of disease. Therefore, these patients have been selected to start anti-TNF therapy at a peak of disease activity. By regression to the mean, one might expect the rate of hospitalizations for disease flares to decrease regardless of treatment. It would have been of interest to have included a cohort of patients with active disease starting an alternative nonbiologic DMARD as a further comparison, to see if a similar pattern emerges. Because other exposures during this time were not controlled, changes in other therapies may also have influenced outcomes.

A similar argument could be made in the observation of the infection rate. Patients who had been admitted with frequent infections prior to consideration of anti-TNF therapy may have been excluded because of relative contraindications for anti-TNF therapy. Therefore, the rate of infection in patients who did start treatment may have been lower than expected during the time preceding anti-TNF, thus resulting in an apparent increase following the start of therapy. Surveillance bias is also possible: if the physician were already aware of the potential infection risk of anti-TNF therapies, he/she would be more likely to admit a patient for treatment of the infection while on anti-TNF therapy, thus elevating the hospitalization rate for infection.

Prospective observational studies do not completely overcome the challenges of bias and confounding. In routine clinical practice, patients are not randomized to treatment, and confounding by indication may be present. These problems can be mitigated to a certain extent by carefully measuring confounders a priori and accounting for these in the evaluation of risk. Nevertheless, residual confounding and biases may still exist in observational studies. Their greatest strength, however, is that these registries and other large patient collections provide a realistic picture of benefits and risks of the biologic drugs and will contribute toward a more informed discussion between patient and physician.

\footnotetext{
AUDREY LOW, MB, CHB, MRCP; KIMME HYRICH, MD, PhD, FRCPC, Arthritis Research UK Epidemiology Unit, Centre for Musculoskeletal Research, Institute of Inflammation and Repair, The University of Manchester, Manchester Academic Health Science Centre, Oxford Road, Manchester M13 9PT, England. Address correspondence to Dr. Hyrich; E-mail:kimme.hyrich@manchester.ac.uk
}

\section{REFERENCE}

1. Singh JA, Christensen R, Wells GA, Suarez-Almazor ME, Buchbinder R, Lopez-Olivo MA, et al. Biologics for rheumatoid arthritis: An overview of Cochrane reviews. Cochrane Database Syst Rev 2009;7:CD007848.

2. Zink A, Strangfeld A, Schneider M, Herzer P, Hierse F, Stoyanova-Scholz M, et al. Effectiveness of tumor necrosis factor inhibitors in rheumatoid arthritis in an observational cohort study: Comparison of patients according to their eligibility for major randomized clinical trials. Arthritis Rheum 2006;54:3399-407.

3. Hyrich KL, Symmons DP, Watson KD, Silman AJ, British Society for Rheumatology Biologics Register. Comparison of the response to infliximab or etanercept monotherapy with the response to cotherapy with methotrexate or another disease-modifying antirheumatic drug in patients with rheumatoid arthritis: Results

Personal non-commercial use only. The Journal of Rheumatology Copyright (c) 2013. All rights reserved. 
from the British Society for Rheumatology Biologics Register. Arthritis Rheum 2006;54:1786-94.

4. Hetland ML, Christensen IJ, Tarp U, Dreyer L, Hansen A, Hansen IT, et al. Direct comparison of treatment responses, remission rates, and drug adherence in patients with rheumatoid arthritis treated with adalimumab, etanercept, or infliximab: Results from eight years of surveillance of clinical practice in the nationwide Danish DANBIO registry. Arthritis Rheum 2010;62:22-32.

5. Soliman MM, Ashcroft DM, Watson KD, Lunt M, Symmons DPM, Hyrich KL. Impact of concomitant use of DMARDs on the persistence with anti-TNF therapies in patients with rheumatoid arthritis: Results from the British Society for Rheumatology Biologics Register. Ann Rheum Dis 2011;70:583-9.

6. Galloway JB, Hyrich KL, Mercer LK, Dixon WG, Fu B, Ustianowski AP, et al. Anti-TNF therapy is associated with an increased risk of serious infections in patients with rheumatoid arthritis especially in the first 6 months of treatment: Updated results from the British Society for Rheumatology Biologics Register with special emphasis on risks in the elderly. Rheumatology 2010;50:124-31.

7. Listing J, Strangfeld A, Kary S, Rau R, von Hinueber U, Stoyanova-Scholz M, et al. Infections in patients with rheumatoid arthritis treated with biologic agents. Arthritis Rheum 2005;52:3403-12.

8. Grijalva CG, Chen L, Delzell E, Baddley JW, Beukelman T, Winthrop KL, et al. Initiation of tumor necrosis factor-alpha antagonists and the risk of hospitalization for infection in patients with autoimmune diseases. JAMA 2011;306:2331-9.

9. Mercer LK, Green AC, Galloway JB, Davies R, Lunt M, Dixon WG, et al. The influence of anti-TNF therapy upon incidence of keratinocyte skin cancer in patients with rheumatoid arthritis: Longitudinal results from the British Society for Rheumatology Biologics Register. Ann Rheum Dis 2012;71:869-74.

10. Askling J, Baecklund E, Granath F, Geborek P, Fored M, Backlin $\mathrm{C}$, et al. Anti-tumour necrosis factor therapy in rheumatoid arthritis and risk of malignant lymphomas: Relative risks and time trends in the Swedish Biologics Register. Ann Rheum Dis 2009;68:648-53.
11. Wolfe F, Michaud K. The effect of methotrexate and anti-tumor necrosis factor therapy on the risk of lymphoma in rheumatoid arthritis in 19,562 patients during 89,710 person-years of observation. Arthritis Rheum 2007;56:1433-9.

12. Curtis JR, Jain A, Askling J, Bridges SL Jr, Carmona L, Dixon W, et al. A comparison of patient characteristics and outcomes in selected European and U.S. rheumatoid arthritis registries. Semin Arthritis Rheum 2010;40:2-14.

13. Zisman D, Haddad A, Hashoul S, Laor A, Bitterman H, Rosner I, et al. Hospitalizations of patients treated with anti-tumor necrosis factor- $\alpha$ agents - A retrospective cohort analysis. J Rheumatol 2013;40:16-22.

14. Brennan A, Bansback N, Nixon R, Madan J, Harrison M, Watson $\mathrm{K}$, et al. Modelling the cost effectiveness of TNF- $\alpha$ antagonists in the management of rheumatoid arthritis: Results from the British Society for Rheumatology Biologics Registry. Rheumatology 2007;46:1345-54.

15. Bansback N, Marra C, Tsuchiya A, Anis A, Guh D, Hammond T, et al. Using the Health Assessment Questionnaire to estimate preference-based single indices in patients with rheumatoid arthritis. Arthritis Care Res 2007;57:963-71.

16. Curtis JR, Patkar N, Xie A, Martin C, Allison JJ, Saag M, et al Risk of serious bacterial infections among rheumatoid arthritis patients exposed to tumor necrosis factor alpha antagonists. Arthritis Rheum 2007;56:1125-33.

17. Askling J, Dixon W. The safety of anti-tumour necrosis factor therapy in rheumatoid arthritis. Curr Opin Rheumatol 2008; 20:138-44.

18. Strangfeld A, Eveslage M, Schneider M, Bergerhausen HJ, Klopsch $\mathrm{T}$, Zink A, et al. Treatment benefit or survival of the fittest: What drives the time-dependent decrease in serious infection rates under TNF inhibition and what does this imply for the individual patient? Ann Rheum Dis 2011;70:1914-20.

J Rheumatol 2013;40:4-6; doi:10.3899/jrheum.121260 